\title{
Integrated FDI/FTC approach for wind turbines using a LPV interval predictor subspace approach and virtual sensors/actuators
}

\author{
Houda Chouiref ${ }^{1,2}$, Boumedyen Boussaid ${ }^{1,3}$, \\ Mohamed Naceur Abdelkrim', Vicenç Puig' and \\ Christophe Aubrun ${ }^{3}$
}

\begin{abstract}
In order to keep wind turbines connected and in operation at all times despite the occurrence of some faults, advanced fault detection and accommodation schemes are required. To achieve this goal, this paper proposes to use the Linear Parameter Varying approach to design an Active Fault Tolerant Control for wind turbines. This Active Fault Tolerant Control is integrated with a Fault Detection and Isolation approach. Fault detection is based on a Linear Parameter Varying interval predictor approach while fault isolation is based on analysing the residual fault signatures. To include fault-tolerance in the control system (already available in the considered wind turbine case study based on the well known SAFEPROCESS benchmark), the information of the Fault Detection and Isolation approach block is exploited and it is used in the implementation of a virtual actuator and sensor scheme. The proposed Active Fault Tolerant Control is evaluated using fault scenarios which are proposed in the wind turbine benchmark to assess its performance. Results show the effectiveness of the proposed Active Fault Tolerant Control approach in faulty situation.
\end{abstract}

\section{Keywords \\ Linear parameter varying, interval predictor, wind turbine, fault diagnosis, virtual sensor, virtual actuator}

Date received: I December 2020; accepted: I January 202 I

\section{Introduction}

In the last years, wind turbines have become one of the important renewable energy sources. However, they can be affected by some fault scenarios which can lead to unsatisfactory performance in many works $^{1-4}$ theses faults are proposed. To avoid these consequences, fault tolerant control (FTC) strategies should be applied to keep the wind turbine operation and performance even under the case of faulty components or instruments. The development of FTC schemes for wind turbines has been already considered in the literature. The proposed approaches can be divided in two groups: active and passive. The passive approach takes into account the faults during the design of the control law ${ }^{5}$ by considering them as uncertainties. This method is limited to small size fault scenarios and generally the obtained performance even in non-faulty scenarios is reduced. ${ }^{6}$ This makes that the active fault tolerant control approach, that overcomes such limitations, has been largely developed. Active Fault Tolerant Control (AFTC) accommodates the fault using the information provided by a Fault Detection and Isolation (FDI) block. ${ }^{7-9}$ Most of research in AFTC of wind turbines has focused on additive sensor and actuator faults using some type of observer scheme in fault detection. ${ }^{10}$ In case of parametric faults, it is necessary to use some parameter estimation scheme that allows to obtain the new parameters after the fault and

\footnotetext{
'Modeling, Analysis and Control of Systems (MACS) Laboratory, National School of Engineers of Gabes, Gabes University, Gabes, Tunisia

${ }^{2}$ Institut de Robòtica i Informàtica Industrial (CSIC-UPC), Universitat Politècnica de Catalunya C/. Llorens i Artigas 4-6, Barcelona, Spain

${ }^{3}$ Centre de Recherche en Autoamtique de Nancyl (CRAN), University of Lorraine, Nancy, France

Corresponding author:

Boumedyen Boussaid, MACS Laboratory, National School of Engineers of Gabes, University of Gabes, Omar Ibn Khattab street, 6029, Zrig, Gabes, Tunisia.

Email: boumedyen.boussaid@enig.rnu.tn
} 
accommodate/reconfigure the control loop accordingly (see e.g., Simani and Castaldi ${ }^{11}$ )

Regarding the application of FTC to wind turbines, there are many references considering the application FTC in generator ${ }^{12,13}$ and drive train subsystems. ${ }^{14}$ There are less references regarding the application to pitch subsystem despite it has an important role in producing an optimal power. The occurrence of a fault in this actuator causes a pitch position error which can result in an insufficient torque. Consequently, the power can not follow the reference set-point. In this work, the proposed fault tolerant method is applied to the pitch subsystem in the faulty case. Most of the FTC schemes for wind turbines have considered Linear Time Invariant (LTI) models. ${ }^{15}$ However, wind turbines behave as nonlinear systems with complex dynamics specially when considering the aerodynamic part. Thus, the use of LTI models for designing FTC schemes for wind turbines could be insufficient to obtain satisfactory results. To overcome such limitations, recent FTC approaches have been proposed to use directly a nonlinear model $^{11,16,17}$ or some multiple-model scheme. ${ }^{18,19}$

Recently, Linear Parameter Varying (LPV) theory has become a standard formalism ${ }^{20}$ for addressing the problem of control of nonlinear systems using pseudo-linear like methods. Many works have been developed using this approach and are applied to wind turbine systems. Wind turbines can be represented by an LPV model that is scheduled with some variables that define the operating point. For example, in Sloth et al., ${ }^{6}$ the LPV approach is used to design a fault-tolerant gain-scheduling controller.

The contribution of this paper is to propose an integrated FDI/FTC approach for wind turbines that solves the problem of control accommodation in case of actuator and sensor faults as the ones proposed in Zhang, ${ }^{3}$ Odgaard and Stoustrup ${ }^{21}$ and Laouti and Othman ${ }^{4}$ and EL Bakri and Boumhidi ${ }^{1}$ where the actuator faults are again not handled as well as the sensor faults. It seems to be a general trend of these solutions, which might indicate that the sensor faults in the benchmark model are easier to accommodate than the actuator faults. This approach combines LPV modeling and identification, robust FDI based on interval-based subspace approaches and virtual actuator/sensor FTC techniques (see Figure 1). This approach is applied to a wind turbine case study proposed as a FDI/FTC benchmark in Odgaard et al. ${ }^{22}$

This paper is organized as follows: In System description Section, the wind turbine benchmark system is detailed. In Fault detection and isolation Section, the fault diagnosis approach is presented and illustrated using the pitch system. In Fault tolerant control Section, the FTC method based on virtual sensor and actuator is described. Application results Section deals with simulation experiments that illustrate the implementation of the proposed approach using the proposed wind turbine benchmark and the

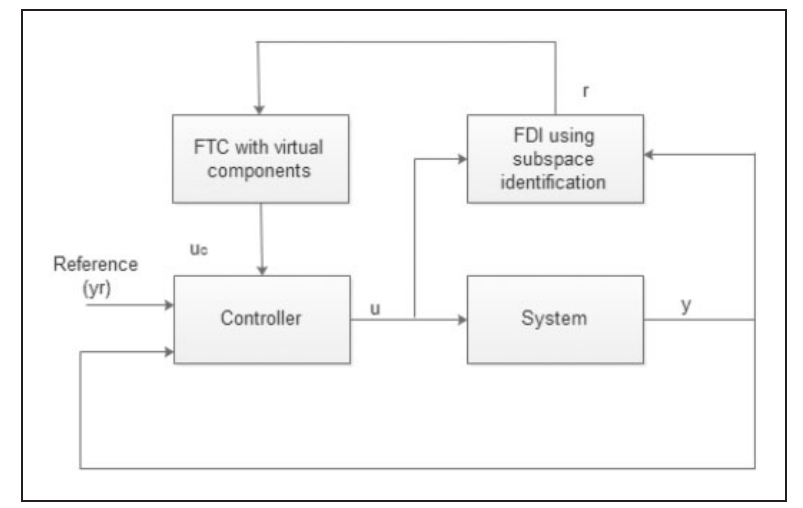

Figure I. Principle of FTC scheme. (a) Pitch measurement and estimation, (b) Pitch residual, (c) Fault indicator.

Table I. Wind turbine parameters.

\begin{tabular}{|c|c|c|c|}
\hline Parameter & Notation & Value & Unit \\
\hline Viscous friction & $B_{g}$ & 47,6 & $\frac{\mathrm{MN} \cdot \mathrm{m} \cdot \mathrm{s}}{\mathrm{rad}}$ \\
\hline Torsion damping & $B_{d t}$ & 775.49 & $\frac{\mathrm{MN} \cdot \mathrm{m} \cdot \mathrm{s}}{\mathrm{rad}}$ \\
\hline Friction coefficient & $B_{r}$ & 7.11 & $\frac{\text { MN.m.s }}{\mathrm{rad}}$ \\
\hline Gear ratio & $N_{g}$ & 95 & \\
\hline Torsion stiffness & $K_{d t}^{0}$ & 2.7 e 9 & $\frac{\mathrm{GN} \cdot \mathrm{m}}{\mathrm{rad}}$ \\
\hline $\begin{array}{l}\text { Efficiency of the } \\
\text { drive train }\end{array}$ & $\eta_{d t}$ & 0.97 & \\
\hline Generator inertia & $J_{g}$ & 390 & $\mathrm{~kg} \cdot \mathrm{m}^{2}$ \\
\hline Rotor inertia & $J_{r}$ & 55 e 6 & $\mathrm{~kg} \cdot \mathrm{m}^{2}$ \\
\hline Time constant & $\alpha_{g c}$ & 0.05 e-3 & \\
\hline $\begin{array}{l}\text { Efficiency of } \\
\text { the generator }\end{array}$ & $\eta_{g c}$ & 0.98 & \\
\hline Radius of blades & $R$ & 57.5 & $m^{2}$ \\
\hline Air density & $\rho$ & 1.225 & $\frac{\mathrm{kg}}{\mathrm{m}^{3}}$ \\
\hline Damping ratio & $\zeta$ & 0.6 & $\frac{\mathrm{rad}}{\mathrm{s}}$ \\
\hline Natural frequency & $\omega_{n}$ & II.II & $\frac{\text { rad }}{\mathrm{s}}$ \\
\hline
\end{tabular}

comparison with existing results. Finally, Conclusion Section gives some concluding remarks.

\section{System description}

As discussed in the introduction, the purpose of this paper is to develop an integrated FDI/FTC scheme based on the LPV approach for wind turbines and illustrate it applied to the benchmark case study proposed in Odgaard and Stoustrup. ${ }^{10}$ The parameters of this case of wind turbine in nominal case are given in the Table $1 .^{23}$

The model of the considered wind turbine is composed of four subsystems: Aerodynamics, Pitch, Drive train and Generator which are described in the following subsections.

\section{Aerodynamics}

The wind turbine aerodynamics is modeled as a torque acting on the blades. This torque, $T_{r}$, is given by: ${ }^{22}$ 


$$
T_{r}(t)=\frac{\rho \pi R^{3} C_{q}\left(\lambda(t), \beta_{j}(t)\right) v_{w}(t)^{2}}{6}
$$

where $\lambda$ is the tip speed ratio, $\rho$ is the density of the air, $R$ is the radius of blades, $v_{w}$ is the wind speed, $\beta_{j}$ is the angle of the pitch of the blade $j$ and $C_{q}$ is the coefficient of the torque.

\section{Drive train}

The drive train is modeled with a flexible two mass system and is used to increase the speed from rotor to generator. The model of the drive train is given as: ${ }^{6}$

$$
\left[\begin{array}{l}
\dot{w}_{r}(t) \\
\dot{w}_{g}(t) \\
\dot{\theta}_{\Delta}(t)
\end{array}\right]=A_{d}\left[\begin{array}{c}
w_{r}(t) \\
w_{g}(t) \\
\theta_{\Delta}(t)
\end{array}\right]+B_{d}\left[\begin{array}{c}
T_{r}(t) \\
T_{g}(t)
\end{array}\right]
$$

where $w_{r}, w_{g}, \theta_{\Delta}, T_{r}, T_{g}$ are respectively the rotor speed, the generator speed, the torsion angle, the rotor and generator torque.The system matrices in (2) are given by

$$
\begin{gathered}
A_{d}=\left[\begin{array}{ccc}
-\frac{B_{d t}+B_{r}}{J_{r}} & \frac{B_{d t}}{N_{g} J_{r}} & -\frac{K_{d t}}{J_{r}} \\
\frac{\eta_{d t} B_{d t}}{N_{g} J_{g}} & \frac{-\frac{\eta_{d t}+B_{d t}}{N_{g}^{2}}-B_{g}}{J_{g}} & \frac{\eta_{d t} K_{d t}}{N_{g} J_{g}} \\
1 & \frac{-1}{N_{g}} & 0
\end{array}\right] \text { and } \\
B_{d}=\left[\begin{array}{cc}
\frac{1}{J_{r}} & 0 \\
0 & -\frac{1}{J_{g}}
\end{array}\right]
\end{gathered}
$$

\section{Generator}

The dynamics of the converter is modeled by a first order transfer function ${ }^{22}$

$$
\frac{T_{g}(s)}{T_{r g}(s)}=\frac{\alpha_{g c}}{s+\alpha_{g c}}
$$

The generator produces a power which is given by

$$
P_{g}(t)=\eta_{g c} w_{g}(t) T_{g}(t)
$$

Pitch

The hydraulic pitch in this benchmark wind turbine is a piston servo mechanism which can be modeled by a second order transfer function as follows: ${ }^{22}$

$$
\frac{\beta(s)}{\beta_{r}(s)}=\frac{\omega_{n}^{2}}{s^{2}+2 \zeta \omega_{n} s+\omega_{n}^{2}}
$$

where $\beta_{r}$ corresponds to the reference values of pitch angles, $w_{n}$ and $\xi$ are respectively the natural frequency and the damping ratio. These parameters are variable with hydraulic pressure $P{ }^{22}$ Then, the representation of the pitch system can be represented by means of the following LPV model which combine the normal and the faulty operation mode according to Sloth et al. ${ }^{6}$ using $P$ as the scheduling variable $\mu$ :

$$
\begin{aligned}
& x(k+1)=A(\mu) x(k)+B(\mu) u(k) \\
& y(k)=C x(k)
\end{aligned}
$$

with

$$
\begin{aligned}
& A(\mu)=\left[\begin{array}{cc}
1 & T_{e} \\
-T_{e} w_{n}^{2}(\mu) & -2 T_{e} \xi(\mu) w_{n}(\mu)+1
\end{array}\right] \text { and } \\
& B(\mu)=\left[\begin{array}{c}
0 \\
T_{e} w_{n}^{2}(\mu)
\end{array}\right]
\end{aligned}
$$

with $T_{e}=0.001$, The state vector includes the pitch angular speed and the position and the output measurement is the angle.

\section{Fault detection and isolation}

In the proposed AFTC presented in Figure 1, the FDI module is based on passive robust approach that combines the LPV subspace estimation approach proposed in Van Willem and Verhagen ${ }^{24}$ and the interval predictor approach introduced in Puig. ${ }^{25}$

\section{Interval predictor approach}

This approach is used to consider the modelling uncertainty and allow to introduce robustness in the fault detection block. The uncertainty in the model is assumed to be concentrated in the parameters that are assumed unknown but bounded by a set $\Theta$ that is described by a zonotope:

$$
\Theta=\theta^{0} \oplus H B^{n}=\left\{\theta^{0}+H z: z \in B^{n}\right\}
$$


where $\theta^{0}$ is the nominal model, $H$ is matrix uncertainty shape, $B^{n}$ is a unitary box composed of $n$ unitary interval vectors $(B=[-1,1])$ and $\oplus$ is the Minkowski sum. In this paper, a particular parameter set $\Theta$ which is bounded by an interval box is used:

$$
\Theta=\left[\underline{\theta_{1}}, \overline{\theta_{1}}\right] \times \ldots\left[\underline{\theta}_{i}, \bar{\theta}_{i}\right] \times \ldots\left[\underline{\theta}_{n_{\theta}}, \bar{\theta}_{n_{\theta}}\right]
$$

where $\underline{\theta}_{i}=\theta_{i}^{0}-\lambda_{i}$ and $\bar{\theta}_{i}=\theta_{i}^{0}+\lambda_{i}$ with $\lambda_{i} \geq 0$ and $i=1, \ldots, n_{\theta}$. The interval box can be represented by means of a zonotope with center $\theta^{0}$ and $H$ equal to an $n_{\theta} \times n_{\theta}$ diagonal matrix:

$$
\begin{aligned}
& \theta^{0}=\left(\frac{\overline{\theta_{1}}+\underline{\theta} 1}{2}, \frac{\bar{\theta}_{2}+\underline{\theta} 2}{2}, \ldots, \frac{\bar{\theta}_{n_{\theta}}+\underline{\theta} n_{\theta}}{2}\right) \\
& H=\operatorname{diag}\left(\lambda_{1}, \lambda_{2}, \ldots, \lambda_{n_{\theta}}\right)
\end{aligned}
$$

The model can be described by the following regressor form:

$$
y(k)=\phi(k) \theta(k)+e(k)
$$

where $\phi(k)$ is the regressor vector that is function of inputs $u(k)$ and outputs $y(k), \theta(k) \in \Theta$ is the parameter vector, $\Theta$ is the set of parameters and $e(k)$ is the noise term assumed unknown but bounded $|e(k)| \leq \sigma$.

Using this approach, the measured data in faultfree case are covered by the interval predicted output.

$$
y(k) \in[\underline{\hat{y}(k)}-\sigma, \overline{\hat{y}(k)}+\sigma]
$$

where

$$
\begin{aligned}
& \underline{\hat{y}(k)}=\hat{y}^{0}(k)-\|\phi(k) H\|_{1} \\
& \overline{\hat{y}(k)}=\hat{y}^{0}(k)+\|\phi(k) H\|_{1}
\end{aligned}
$$

and $\hat{y}^{0}(k)$ is the prediction with nominal parameters with $\theta^{0}=\left[\theta_{1}, \theta_{2}, \ldots, \theta_{n_{\theta}}\right]^{T}$ obtained using the parameter estimation method presented here after

$$
\hat{y}^{0}(k)=\phi(k) \theta^{0}
$$

\section{Estimation approach}

Problem formulation. to estimate the nominal LPV parameters in (8) using the subspace method proposed in Van Willem and Verhagen ${ }^{24}$ this model is expressed as follows:

$$
x(k+1)=\sum_{i=1}^{m} \mu^{(i)}(k)\left(A^{(i)} x(k)+B^{(i)} u(k)+K^{(i)} e(k)\right)
$$

$$
y(k)=C x(k)+D u(k)+e(k)
$$

where $u(k) \in R^{r}, x(k) \in R^{n}, y(k) \in R^{l}$ are the input, state and output vectors over time $k=\{1, \ldots, N\}$ and the noise process $e(k)$ is a zero-mean white sequence and $m$ is the number of scheduling parameters (or local models) that are supposed to be measurable in real time. The vector of scheduling parameters is defined as follows:

$$
\mu(k)=\left[1, \mu^{(2)}(k), \ldots, \mu^{m}(k)\right]^{T}
$$

Then, equations (17) and (18) can be rewritten in the predictor form as:

$$
x(k+1)=\sum_{i=1}^{m} \mu^{(i)}(k)\left(\tilde{A}^{(i)} x(k)+\tilde{B}^{(i)} u(k)+K^{(i)} y(k)\right)
$$

with

$$
\begin{aligned}
& \tilde{A}^{(i)}=A^{(i)}-K^{(i)} C \\
& \tilde{B}^{(i)}=B^{(i)}-K^{(i)} D
\end{aligned}
$$

Problem solution. Defining $z(k)=\left[u(k)^{T}, y(k)^{T}\right]^{T}, X=$ $[x(p+1), \ldots, x(N)]$ and

$$
\bar{z}^{p}(k)=\left[\begin{array}{c}
z(k) \\
z(k+1) \\
\cdot \\
\cdot \\
\cdot \\
z(k+p-1)
\end{array}\right]
$$

where $p$ is a past window length. By introducing the matrix

$$
P_{p / k}=\mu(k+p-1) \otimes \ldots \otimes \mu(k) \otimes I_{r+l}
$$

we can define

$$
N_{k}^{p}=\left[\begin{array}{cccc}
P_{p / k} & \cdot & \cdot & 0 \\
\cdot & P_{p-1 / k+1} & & \\
\cdot & & \cdot & \\
\cdot & & & \cdot \\
0 & & & P_{1 / k+p-1}
\end{array}\right]
$$

Then, the matrices $U, Y$ and $Z$ are defined as follows

$$
U=[u(p+1), \ldots, u(N)]
$$




$$
\begin{aligned}
& Y=[y(p+1), \ldots, y(N)] \\
& Z=\left[N_{1}^{p} \bar{z}^{p}(1), \ldots, N_{N-p+1}^{p} \bar{z}^{p}(N-p+1)\right]
\end{aligned}
$$

The controllability matrix can be expressed as:

$$
\kappa^{p}=\left[l_{p}, \ldots, l_{1}\right]
$$

with

$$
l_{1}=\left[\bar{B}^{(1)}, \ldots, \bar{B}^{(m)}\right]
$$

and

$$
l_{j}=\left[\tilde{A}^{(1)} l_{j-1}, \ldots, \tilde{A}^{(m)} l_{j-1}\right]
$$

with $\bar{B}^{(i)}=\left[\tilde{B}^{(i)}, K^{(i)}\right]$

If the matrix $\left[Z^{T}, U^{T}\right]$ has full row rank, we can estimate the matrices $C \kappa^{p}$ and $D$ by solving the next linear regression problem $^{26}$

$$
\min _{C \kappa^{p}, D}\left\|Y-C \kappa^{p} Z-D U\right\|_{F}^{2}
$$

where $\|\cdot\|_{F}$ is the Frobenius norm. As in the case of LTI system identification, this problem can be solved for the considered type of LPV systems by using traditional least square methods. ${ }^{27}$

According to literature, ${ }^{28,29}$ if the matrix $\left[\begin{array}{ll}Z^{T} & U^{T}\end{array}\right]$ has full row rank, the equation (23) has a unique solution and is given by

$$
\left[\begin{array}{ll}
\hat{C \kappa^{p}} & \hat{D}
\end{array}\right]=Y\left[\begin{array}{ll}
Z^{T} & U^{T}
\end{array}\right]\left(\left[\begin{array}{l}
Z \\
U
\end{array}\right]\left[\begin{array}{ll}
Z^{T} & U^{T}
\end{array}\right]\right)^{-1}
$$

When $p$ is large, the solution is computed by using the SVD of the matrix:

$$
\left[\begin{array}{l}
Z \\
U
\end{array}\right]=\left[\begin{array}{ll}
v & v_{\perp}
\end{array}\right]\left[\begin{array}{cc}
\sum_{m} & 0 \\
0 & 0
\end{array}\right]\left[\begin{array}{c}
V^{T} \\
V_{\perp}^{T}
\end{array}\right]
$$

The solution obtained when minimizing the norm is given by:

$$
\left[\begin{array}{ll}
\hat{C \kappa}^{p} & \hat{D}
\end{array}\right]=Y V \sum_{m}^{-1} v^{T}
$$

To evaluate the performance of the identified system, we use the Variance-Accounted-For (VAF) value which is defined as:

$$
\begin{aligned}
& \operatorname{VAF}(y(k), \hat{y}(k)) \\
& =\max \left\{1-\frac{\operatorname{var}(y(k)-\hat{y}(k))}{\operatorname{var}(y(k))}, 0\right\} \times 100 \%
\end{aligned}
$$

where $\operatorname{var}()$ denotes the variance, $\hat{y}(k)$ and $y(k)$ are respectively the estimated and real system outputs.

\section{Parameter uncertainty estimation}

Once the nominal LPV parameters in (8) have been obtained using the subspace method recalled in previous section, to characterize the parameter set $\Theta$, defined in (9) consistent with the data collected in a fault-free scenario, the bounds (11) should be obtained. The uncertain parameter estimation algorithm proceeds by solving the following optimization problem:

$$
\begin{aligned}
& \min \sum_{i=1}^{n_{\theta}} \lambda_{i}^{2} \\
& \text { subject to : } \\
& \quad y_{i}(k) \in\left[\hat{y}_{i}(k)-\sigma_{i}, \overline{\hat{y}}_{i}(k)+\sigma_{i}\right] \\
& \overline{\hat{y}}(k)=\hat{y}^{0}(k)+\|\phi(k) H\|_{1} \\
& \hat{y}(k)=\hat{y}^{0}(k)-\|\phi(k) H\|_{1} \\
& \hat{\hat{y}}^{0}(k)=\phi(k) \theta^{0} \\
& \quad H=\operatorname{diag}\left(\lambda_{1}, \lambda_{2}, \ldots, \lambda_{n_{\theta}}\right) \\
& \quad \text { For all } i=1, \ldots, n_{y} \text { and } k=1, \ldots, N
\end{aligned}
$$

To summarize, the proposed uncertain LPV subspace identification approach is described in Algorithm 1.

Algorithm 1 LPV uncertain parameter estimation

1: Create the matrices $U$ and $Z$ using (20).

2: Obtain the SVD decomposition (25) of $U$ and $Z$.

3: Solve the linear problem in (23) using (26) and obtain nominal parameters $\theta^{0}$

4: Estimate the parameter uncertain bounds solving the optimization problem (28)

\section{Fault isolation scheme}

The fault isolation will be designed considering the set of faults proposed in the wind turbine benchmark case study ${ }^{30}$ that are described in the following taking into account their type:

- Sensor faults. The first sensor fault scenario considered affects the pitch position measurements, which is denoted as $\Delta \beta_{m}$. The second fault scenario corresponds to the rotor speed measurement and is denoted as $\Delta w_{g, m}$ and $\Delta w_{r, m}$. These faults are either electrical or mechanical faults, and can be classified as a fixed value or a gain factor on the measurements. These sensor faults cause a false measurement that affects the performance of the control law.

- Actuator faults. Actuator faults may occur in the pitch actuator or in the converter. In the first subsystem, faults results in a change of dynamics either due to dropped pressure or high air content 
Table 2. Faults affecting benchmark system.

\begin{tabular}{llll}
\hline No & Fault type & Symbol & Type \\
\hline$f_{1}$ & Sensor & $\Delta \beta_{1, m l}$ & Fixed values \\
$f_{2}$ & Sensor & $\Delta \beta_{2, m l}$ & Gain factor \\
$f_{3}$ & Sensor & $\Delta w_{r, m l}$ & Fixed values \\
$f_{4}$ & Sensor & $\Delta w_{r, m 2}$ & Gain factor \\
$f_{5}$ & Sensor & $\Delta w_{g, m l}$ & Fixed values \\
$f_{6}$ & Sensor & $\Delta w_{g, m 2}$ & Gain factor \\
$f_{7}$ & Actuator & $\Delta T_{g}$ & Offset \\
$f_{8}$ & Actuator & $\Delta \beta$ (hydraulic) & Dynamic change \\
$f_{9}$ & Actuator & $\Delta \beta($ Air in oil) & Dynamic change \\
$f_{10}$ & System & $\Delta w_{g}, \Delta w_{r}$ & Dynamic change \\
\hline
\end{tabular}

in the oil. These faults are denoted as $\Delta \beta$. In the second subsystem, faults manifests through an additive offset. The cause of this fault is an offset in the internal converter control loop. These faults are denoted as $\Delta T_{g}$. Faults in actuator of the wind turbine caused slow control actions.

- System faults. The considered system faults occur in the drive train, where the friction changes with time. These faults are denoted as $\Delta w_{r}$ and $\Delta w_{g}$. This type of fault is not that severe, but it is an indication of the wear of the drive train, which finally results in its total breakdown. This system fault increases the level of drive train vibrations.

All these faults are summarized and listed in Table 2. Since the control system can be sensitive to different type of faults, a fault isolation is needed to localize the type of fault occurring in the wind turbine.

Using the sensors available in the wind turbine benchmark and the model presented in Section 2, the following residuals can be obtained applying structural analysis and the perfect matching algorithm $^{8}$

$$
\begin{aligned}
& r_{1}(k)=w_{r m 1}(k)-w_{r m 2}(k) \\
& r_{2}(k)=w_{r m 2}(k)-\widehat{w}_{r m 2}(k) \\
& r_{3}(k)=w_{g m 1}(k)-w_{g m 2}(k) \\
& r_{4}(k)=w_{g m 2}(k)-\widehat{w}_{g m 2}(k) \\
& r_{5}(k)=\beta_{1 m 1}(k)-\beta_{1 m 2}(k) \\
& r_{6}(k)=\beta_{1 m 2}(k)-\widehat{\beta}_{1 m 2}(k) \\
& r_{7}(k)=\beta_{2 m 1}(k)-\beta_{2 m 2}(k) \\
& r_{8}(k)=\beta_{2 m 2}(k)-\widehat{\beta}_{2 m 2}(k) \\
& r_{9}(k)=\beta_{3 m 1}(k)-\beta_{3 m 2}(k) \\
& r_{10}(k)=\beta_{3 m 2}(k)-\widehat{\beta}_{3 m 2}(k) \\
& r_{11}(k)=\tau_{g m}(k)-\hat{\tau}_{g}(k) \\
& r_{12}(k)=P_{g m}(k)-\eta_{g} w_{g m 2}(k) \tau_{g m}(k)
\end{aligned}
$$

Table 3. Fault signature matrix.

\begin{tabular}{lcccccccccc}
\hline & $f_{1}$ & $f_{2}$ & $f_{3}$ & $f_{4}$ & $f_{5}$ & $f_{6}$ & $f_{7}$ & $f_{8}$ & $f_{9}$ & $f_{10}$ \\
\hline$R_{1}$ & & & $\times$ & $\times$ & & & & & & \\
$R_{2}$ & $\times$ & $\times$ & & & & & $\times$ & $\times$ & $\times$ & $\times$ \\
$R_{3}$ & & & & & $\times$ & $\times$ & & & & \\
$R_{4}$ & $\times$ & $\times$ & & & & & $\times$ & $\times$ & $\times$ & $\times$ \\
$r_{5}$ & $\times$ & & & & & & & & & \\
$r_{6}$ & $\times$ & & & & & & & $\times$ & & \\
$r_{7}$ & & $\times$ & & & & & & & & \\
$r_{8}$ & & $\times$ & & & & & & & $\times$ & \\
$r_{9}$ & & & & & & & & & & \\
$r_{10}$ & & & & & & & & & & \\
$r_{11}$ & & & & & & & $\times$ & & & \\
$r_{12}$ & & & & & & & $\times$ & & & $\times$ \\
\hline
\end{tabular}

Then, fault detection will be based on checking if (13) is satisfied. In case that, it is not satisfied a fault can be indicated. Otherwise, nothing can be said. It is carried out on the basis of fault signatures, generated by the detection module, and their relation with all the considered faults, $f(k)=\left\{f_{a}(k), f_{y}(k)\right\}$. The result of the fault detection test (13) allows obtaining a set of fault signatures $\psi(k)=\left[\psi_{1}(k), \psi_{2}(k), \ldots, \psi_{n y}(k)\right]$, where each fault indicator is given by:

$$
\psi_{i}(k)=\left\{\begin{array}{lll}
0 & \text { if } & y(k) \in[\underline{\hat{y}(k)}-\sigma, \overline{\hat{y}(k)}+\sigma] \\
1 & \text { if } & y(k) \notin[\underline{\hat{y}(k)}-\sigma, \overline{\hat{y}(k)}+\sigma]
\end{array}\right.
$$

Then, the binarized residuals are matched against the fault signature denoted as $M$ and presented in Table 3. An element $m_{i, j}$ of $M$ is equal to 1 if the fault $f^{j}$ affects the computation of the residual $r_{i}$; otherwise $m_{i, j}=0$. A column of $M$ is known as a theoretical fault signature and indicates which residuals are affected by a given fault.

The procedure accepted as standard by the FDI community involves finding a matching between the observed fault signature and one of the theoretical fault signatures. However, this reasoning is not appropriate for variables varying in the time but bounded. Due to the uncertainty, when a fault is present in the system, an undefined number of the residuals affected by the fault can be found inconsistent, mainly depending on the sensitivity of each residual with respect to the fault and on the fault magnitude. In other words, the observed fault signature will not exactly match the theoretical signature of the present fault. In this case, if the column-matching procedure is used, then the particular fault will not be identified. An appropriate reasoning should only consider the residuals that are inconsistent when searching for the fault (inconsistency is relevant, consistency is not). A residual that is found inconsistent indicates that one of the faults that affect the residual is acting on the system. But the contrary is not true, if a 
residual is satisfied, it does not assure that none of the associated faults is present. According to the established terminology, the used algorithm must avoid single-fault exoneration (which is implicit in the column matching reasoning). Under single-fault assumption, this can be easily achieved by taking into account that the fault that is actually present in the system has to affect all the residuals that have been found inconsistent according to the observed fault signature (if not, the single fault hypothesis can not explain the observed behavior). Algorithm 2 summarizes an isolation procedure based on this idea.

Algorithm 2 fault detection and isolation

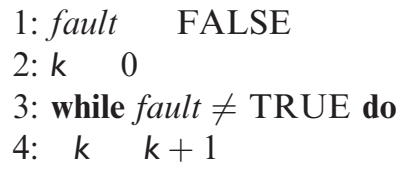

5: Obtain input-output data $\{u(k), y(k)\}$ at time instant $k$

6: Compute $\hat{y}(k)$ using (17)-(18) for each submodel in residual expressions (29) with the parameters adjusted with the parameter estimation algorithm in Section 3.2

7: $\quad$ for $i=1$ to $n_{y}$ do

8: $\quad$ if $y_{i}(k) \in\left[\underline{\hat{y}_{i}(k)}-\sigma, \overline{\hat{y}_{i}(k)}+\sigma\right]$ then

9:

10:

11:

12:

13:

14

15: $\mathcal{F} \mathcal{C} \leftarrow\left\{f^{1}, f^{2}, \ldots, f^{n_{f}}\right\}$

16: for $i=1$ to $n_{y}$ do

17: if $\psi_{i k}=1$ then

18: $\quad$ for $j=1$ to $n_{f}$ do

19: $\quad$ if $m_{i, j}=0$ then

20: $\quad \mathcal{F C} \leftarrow \mathcal{F C}-f^{j}$

21: $\quad$ end if

22: $\quad$ end for

23: $\quad$ end if

24: end for

25: end while

26: Fault candidate set $\mathcal{F C}$

\section{Fault tolerant control}

\section{Introduction}

In terms of control, the wind turbine works in two regions: At low wind speeds, in the partial load region, the turbine is controlled to maximize the power output that is achieved by adjusting the generator torque to obtain an optimum ratio between the wind speed and the tip speed of the blades which is considered in Kamal and Aitouche. ${ }^{31}$ At higher wind speeds, in the full load region, the wind turbine is controlled to reduce loads by producing a rated power output at a constant rotor speed, which is obtained by pitching the blades to adjust the efficiency of the rotor. ${ }^{11}$ In this paper, the problem of control by pitching the blade will be treated applying a constant generator torque where the speed or a power PI controller can be used depending on the operating region. The LPV theory has already been proposed for designing controller in non faulty case. But, recently, it has also been used for FTC design. ${ }^{32-35}$

In this work, fault tolerance has been included in the wind turbine control system by designing virtual sensors and actuators using the LPV model of the wind turbine. The goal of the FTC strategy, based on virtual sensors/actuactors, is to hide the fault to the controller to obtain the nominal property. When a fault occurs, this approach is based on inserting a block between the faulty plant and the nominal controller to allow the controller to see the plant like is in the non faulty case. This block can be a virtual actuator or virtual sensor according to the fault type. Using this method with virtual components, the nominal controller is kept and only the faulty plant will be reconfigured. In actuator faulty case, the new signal control is obtained from the output of the nominal controller considering the fault scenario. In case of virtual sensors, the reconfiguration block uses the faulty plant output as input and compute the fault free one. This hiding approach have many advantages: ${ }^{36}$

- Whatever the nominal regulator, this method can be used.

- In the case of partial fault effect, using this design, it is not necessary to redesign the controller.

- In the case of a human operator, this design reduces its efforts and stress.

\section{LPV virtual sensor/actuator}

In the following, a simplified explanation of virtual sensor and actuator is provided (for a more detailed description, see Blanke et al. ${ }^{8}$ )

Virtual actuator. From the conceptual point of view, the idea is that by means of the virtual actuator block, the transfer function of the faulty plant behaves as in non-faulty situation:

$$
G_{a}(s) G_{f}(s)=G_{n}(s)
$$

where $G_{a}, G_{f}, G_{n}$ are respectively the transfer function of virtual actuator, faulty and nominal plant.

In case of considering the LPV model of the wind turbine, the implementation should be done using the state-space representation

$$
\dot{x}(t)=A(\mu) x(t)+B_{f}(\mu) u(t)
$$




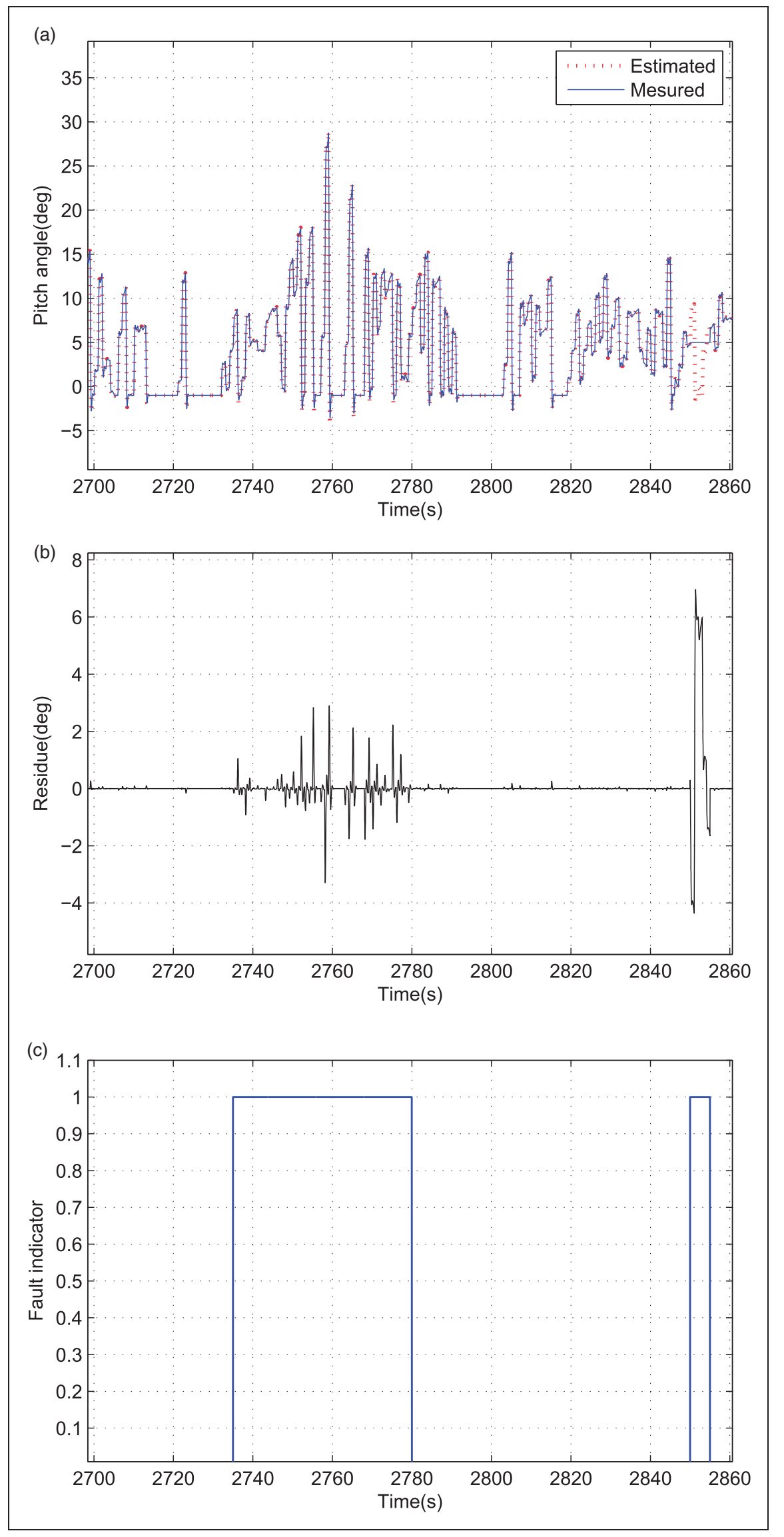

Figure 2. Fault detection results. (a) Pitch measurement and estimation, (b) Error between real and desired signal. 


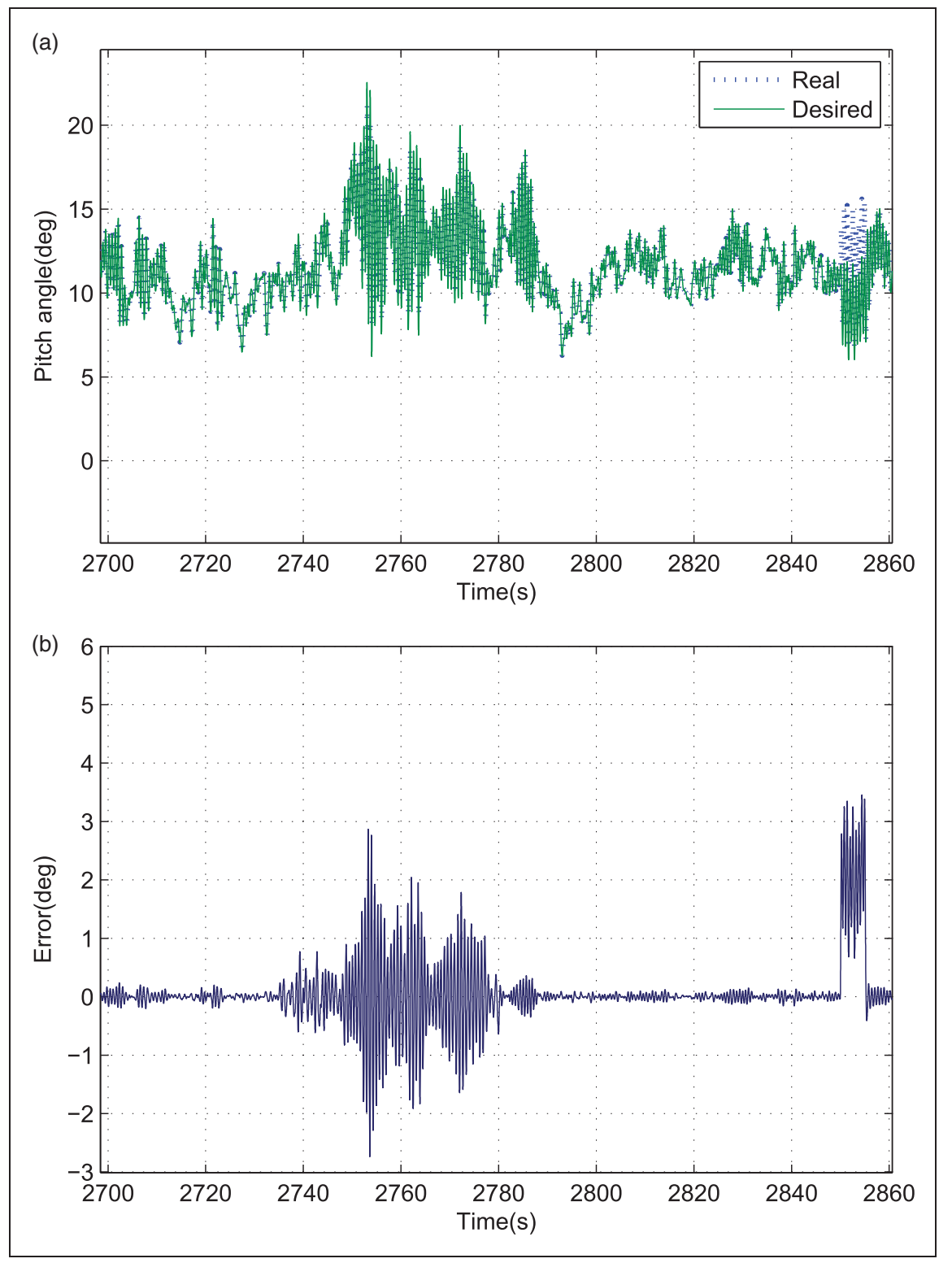

Figure 3. System response in case of fault without FTC.

where $B_{f}=B(\mu) \operatorname{diag}\left(\psi_{1} \ldots \psi_{n u}\right), \psi_{i}$ is the effectiveness of the $i^{\text {th }}$ actuator, $\psi_{i} \in[0,1]$.

If rank $\left(B_{f}\right)=\operatorname{rank}(B)$, the new control signal is obtained by

$$
u_{F T C}(t)=N(\mu) u_{n}(t)
$$

where $u_{n}$ is the output of the controller, $N(\mu)=B_{f}^{+} B$ with $B_{f}^{+}$is the pseudo inverse of $B_{f}$.

Virtual sensor. Considering the output equation in sensor fault case

$$
\begin{aligned}
& \dot{x}_{f}(t)=A(\mu) x_{f}(t)+B u(t) \\
& y_{f}(t)=C_{f}(\gamma) x_{f}(t)+f_{y}(t) \\
& y_{F T C}(t)=P()\left(y_{f}(t)-f_{y}(t)\right)
\end{aligned}
$$

where $C_{f}=\operatorname{diag}\left(\gamma_{1} \ldots \gamma_{n y}\right) C, \gamma_{i}$ is the effectiveness of the $i^{\text {th }}$ sensor, $\gamma_{i} \in[0,1]$.

If $\operatorname{rank}\left(C_{f}(\gamma)\right)=\operatorname{rank}\left(\begin{array}{l}C \\ C_{f}\end{array}\right)$, the new signal is obtained as follows.

$$
y_{F T C}(t)=P(\gamma)\left(y_{f}(t)-f_{y}(t)\right)
$$

where $P=C C_{f}^{+}$with $C_{f}^{+}$is the pseudo inverse of $C_{f}$.

\section{Application to the wind turbine benchmark}

To illustrate the use of the virtual actuator and sensor FTC schemes, the case of the two fault scenarios proposed in the wind turbine benchmark ${ }^{22}$ are used.

Actuator fault. In case of the pitch actuator fault scenario proposed in the wind turbine benchmark, ${ }^{22}$ a change in the parameters $\xi$ and $w_{n}$ of (5) occurs. 


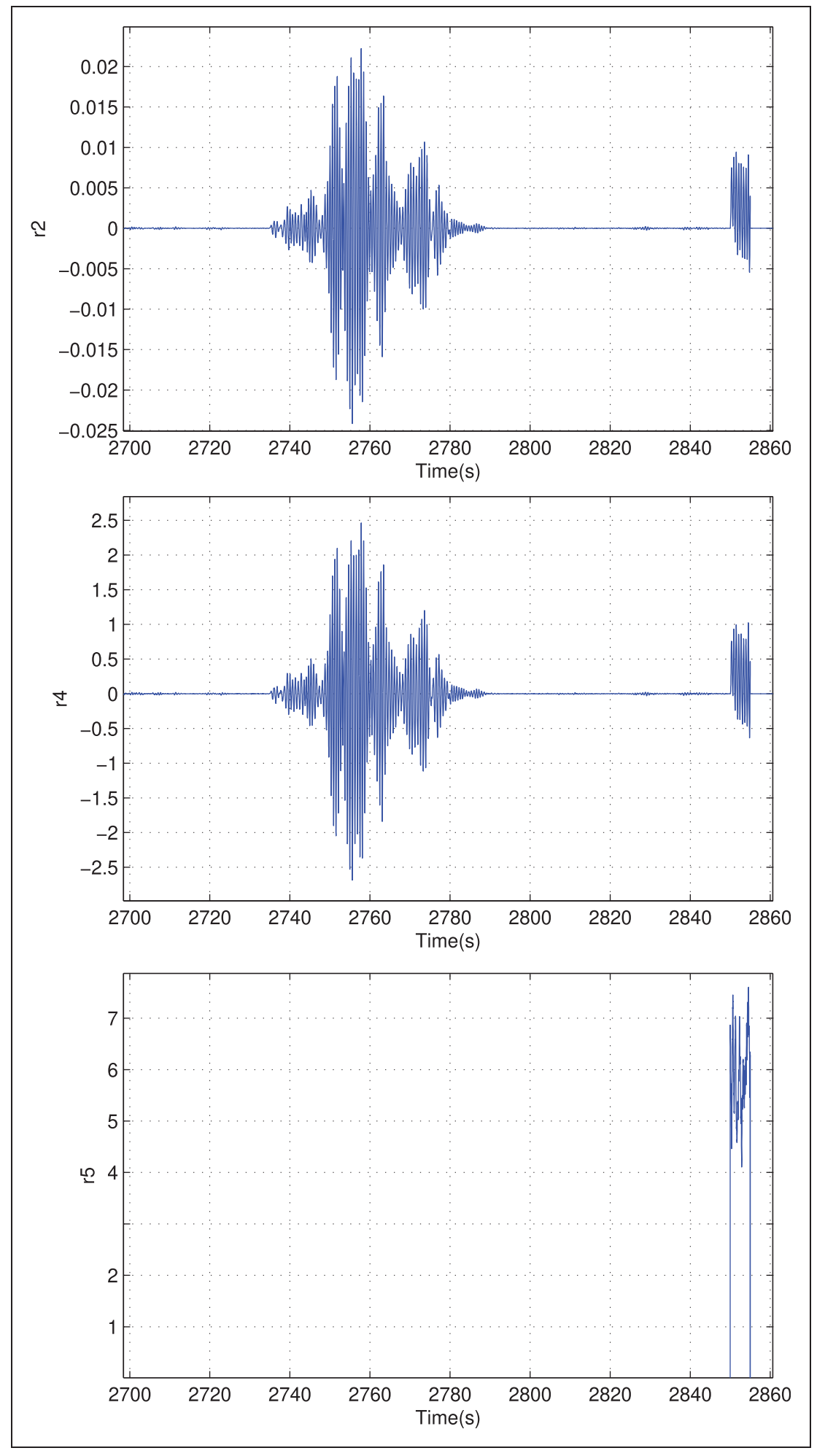

Figure 4. Residual in faulty case.

Then, the virtual actuator can be conceptually designed according to (31) as follows

$$
\begin{aligned}
& G_{a}(s)=G_{f^{-1}}(s) G_{n}(s) \\
& =\frac{w_{n}^{2}\left(s^{2}+2 \hat{\xi}(\mu) \hat{w}_{n}(\mu) s+\hat{w}_{n}(\mu)^{2}\right)}{\hat{w}_{n}(\mu)^{2}\left(s^{2}+2 \xi w_{n} s+w_{n}{ }^{2}\right)}
\end{aligned}
$$

although the design using the LPV model will be done using the state space representation of (5). Then, in case of fault the control signal will be generated as follows:

$$
\beta_{r e f}^{F T C}=\frac{w_{n}^{2}\left(s^{2}+2 \hat{\xi}(\mu) \hat{w}_{n}(\mu) s+\hat{w}_{n}(\mu)^{2}\right)}{\hat{w}_{n}(\mu)^{2}\left(s^{2}+2 \xi w_{n} s+w_{n}^{2}\right)} \beta_{r e f}
$$




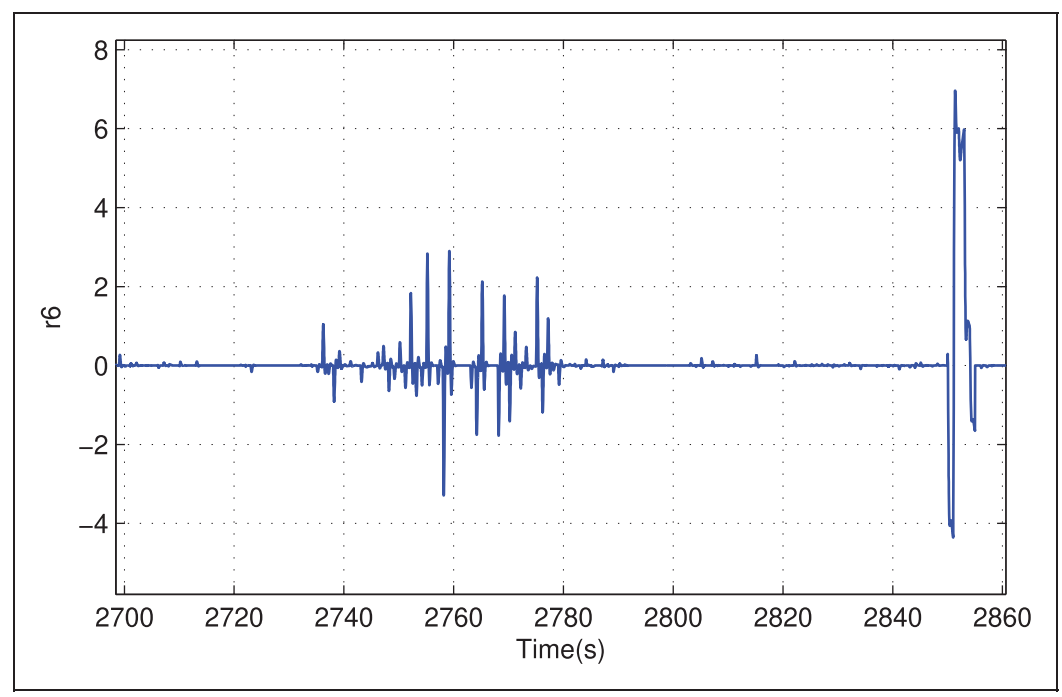

Figure 4. Continued

where $\hat{\xi}(\mu)$ and $\hat{w}_{n}(\mu)$ are respectively the estimated damping ratio and natural frequency obtained by the application of subspace identification method presented in Section 3.2 using the pressure as scheduling variable and in this case two local models are used.

Sensor fault. In the case of a sensor fault scenario proposed in the wind turbine benchmark, ${ }^{22}$ the pitch system input is modified as follows:

$$
\beta_{1 c}=\beta_{1 r e f}+\beta_{1 f}
$$

where

$$
\beta_{1 f}=\beta_{1}-0.5\left(\beta_{1 m 1}+\beta_{1 m 2}\right)
$$

$\beta_{1 \text { ref }}$ is the output of the controller, $\beta_{1}$ is the real pitch angle, $\beta_{1 m 1}$ and $\beta_{1 m 2}$ are the measured pitch angle with two different sensors.

In the case of the complete loss of the sensor, the measurement issued from the faulty sensor is neglected and it is replaced by the measurement issued from the redundant sensor. In the case of partial sensor fault, the virtual sensor, which compensates the fault, generates a new control signal given by:

$$
\beta_{1 F T C}=\beta_{1 r e f}+\beta_{1 f}+\Delta \beta_{1}=\beta_{1 r e f}+\beta_{1}-\beta_{1 m 2}
$$

then

$$
\Delta \beta_{1}=0.5\left(\beta_{1 m 1}-\beta_{1 m 2}\right)
$$

Integration with the FDI scheme. Algorithm 3 summarizes the integration of the FDI and the FTC schemes.
Algorithm 3 FTC algorithm using virtual sensor/ actuator

1: Run the system with nominal controller.

2: If an actuator fault is detected and isolated using Algorithm 2, activate the virtual actuator based on (33) after estimating faulty parameters using subspace identification method presented in Section 3.2

3: If a sensor fault is detected and isolated using Algorithm 2, activate the virtual sensor based on (37) after estimating faulty parameters using subspace identification method presented in Section 3.2

3: Run the accommodated system.

\section{Application results}

The two fault scenarios already considered for presenting the design of the virtual actuator and sensor are used to illustrate the performance of the proposed AFTC approach:

1. Fault scenario 1: Actuator pitch fault between 2735 s and 2780 s (case of fault $f_{8}$ in Table 2).

2. Fault scenario 2: Sensor pitch fault between 2850s and 2855 s (case of fault $f_{1}$ in Table 2).

The measured and the estimated pitch angle in these fault scenarios, the residual calculated as difference between the measured and the estimated pitch signal and the fault indicator are presented in Figure 2.

In the faulty system case, using the nominal controller already available in the wind turbine benchmark system, the desired and the real pitch angle 


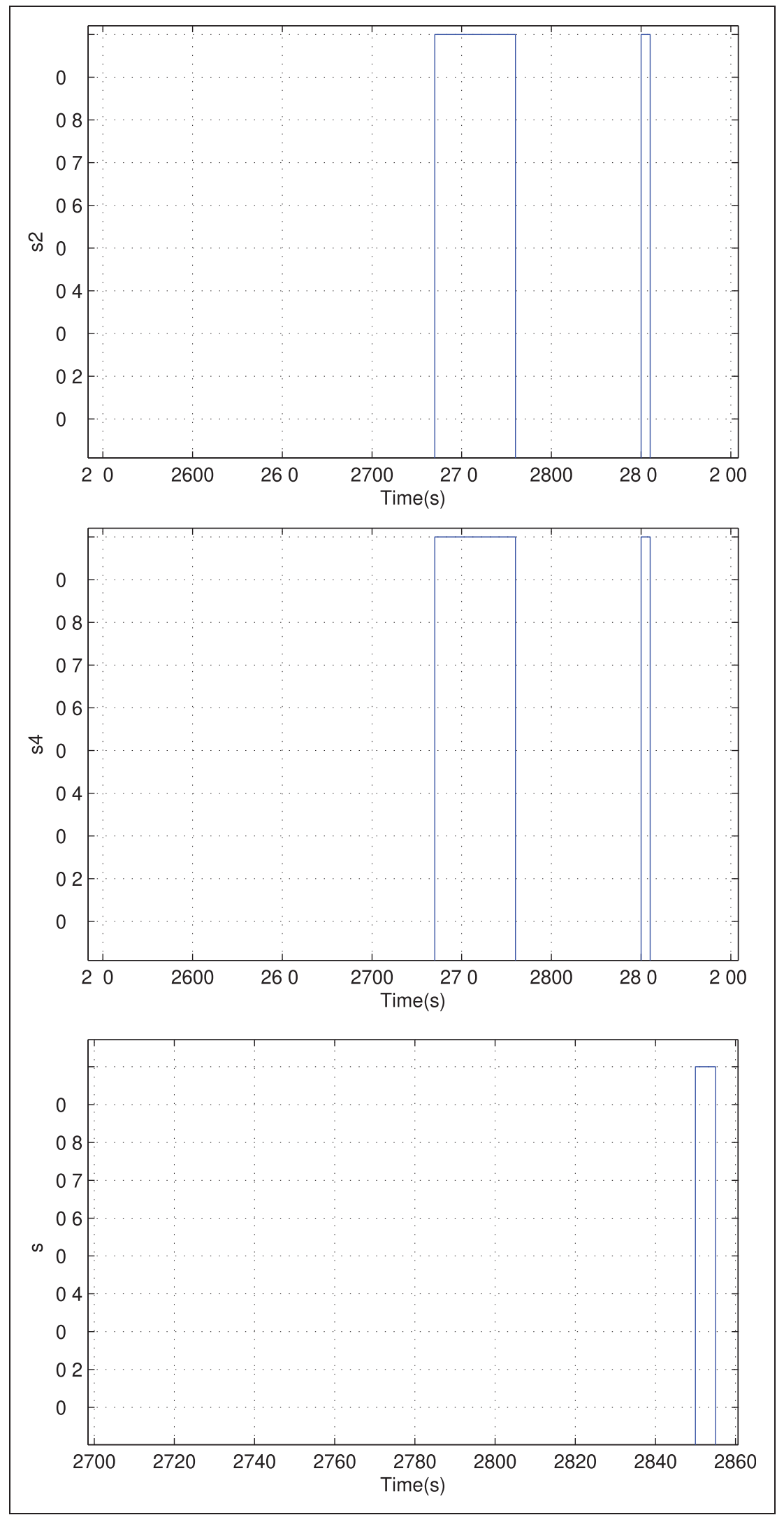

Figure 5. Observed fault signature. (a) Actuator fault indicator $\left(f_{8}\right)$, (b) Sensor fault indicator $\left(f_{1}\right)$. 


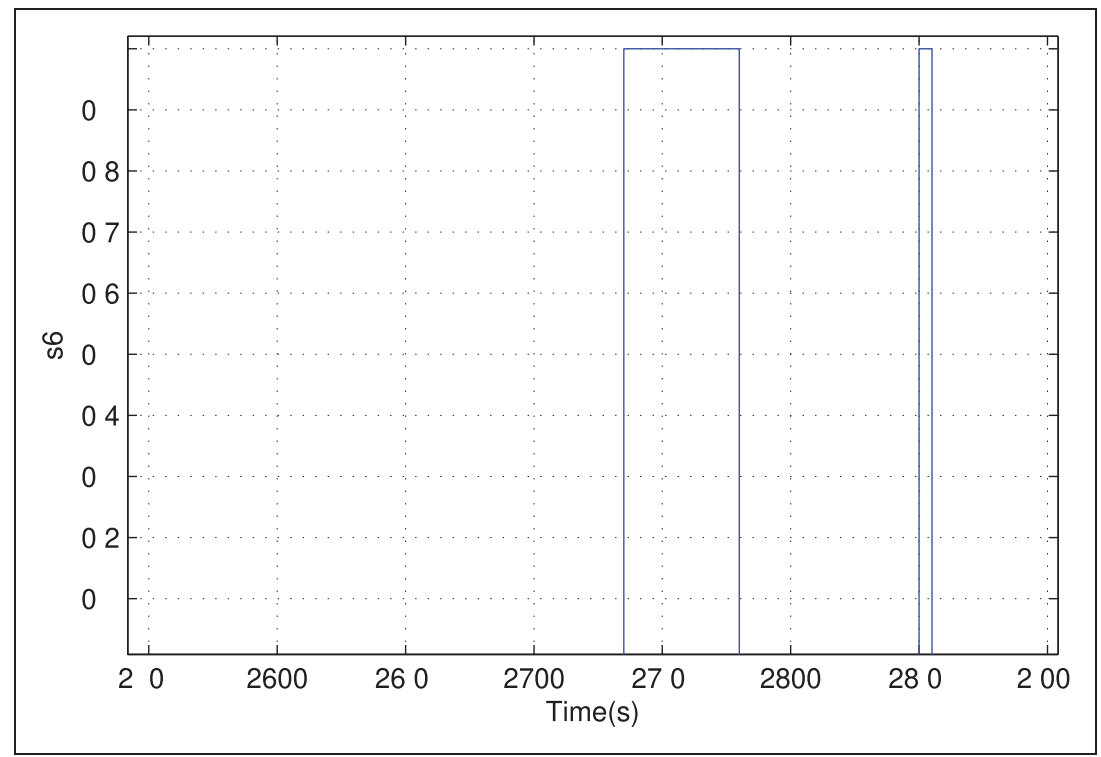

Figure 5. Continued

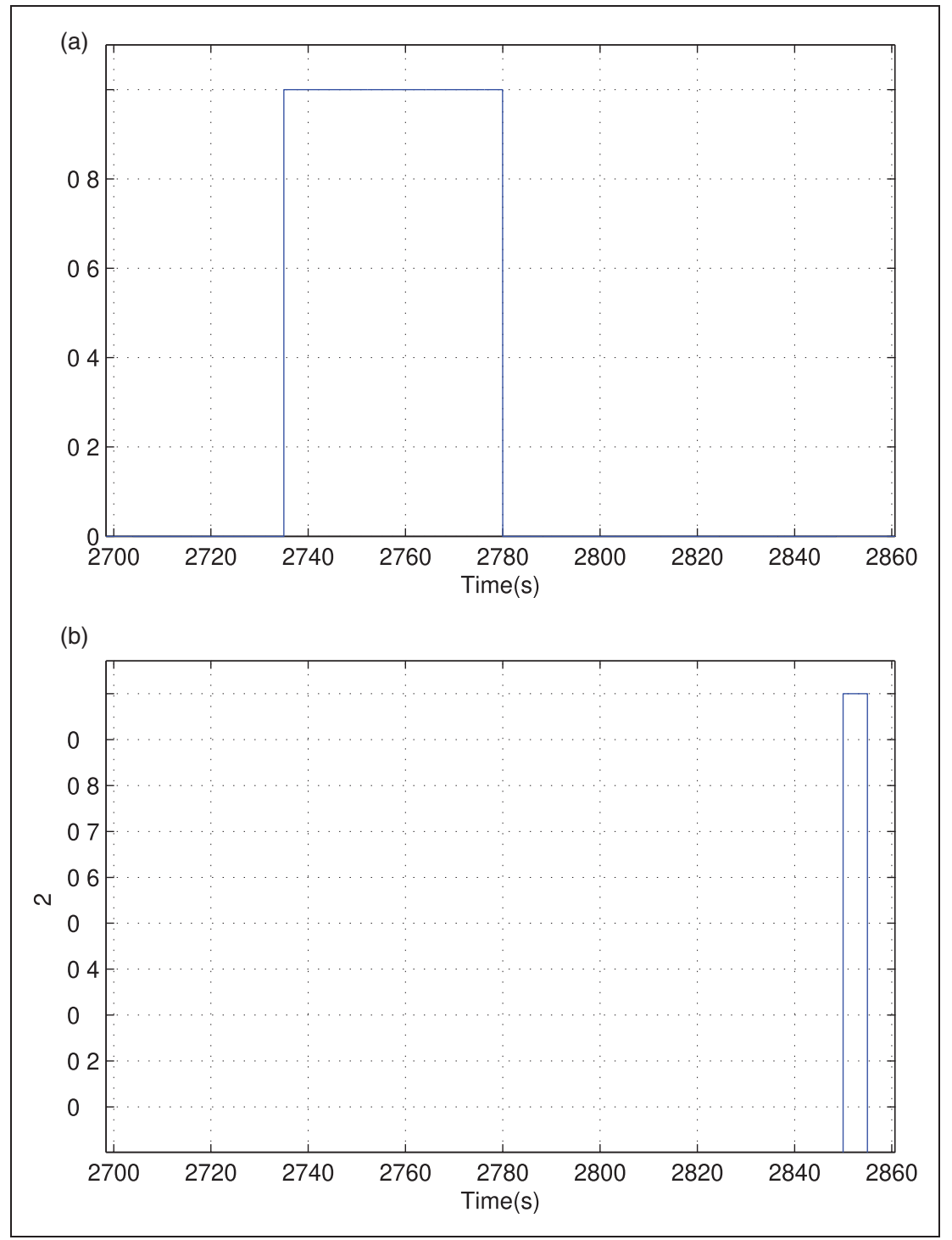

Figure 6. Fault isolation. (a) Damping ratio, (b) Natural frequency 
Table 4. Comparison of some fault detection results.

\begin{tabular}{llllll}
\hline No & Required TD & ATD & TDI & TD2 & TD3 \\
\hline$F_{1}$ & $<10 T s$ & $T s$ & $3 T s$ & $44 T s$ & 6Ts \\
$F_{6}$ & $<10 T s$ & $T s$ & $3 T s$ & $22 T s$ & 3Ts \\
$F_{7}$ & $<3 T s$ & $T s$ & $3 T s$ & $35 T s$ & $3 T s$ \\
$F_{8}$ & $<8 T s$ & $T s$ & $375 T s$ & NC & 2Ts \\
$F_{10}$ & $<50 T s$ & $T s$ & $3 T s$ & & 36Ts \\
\hline
\end{tabular}

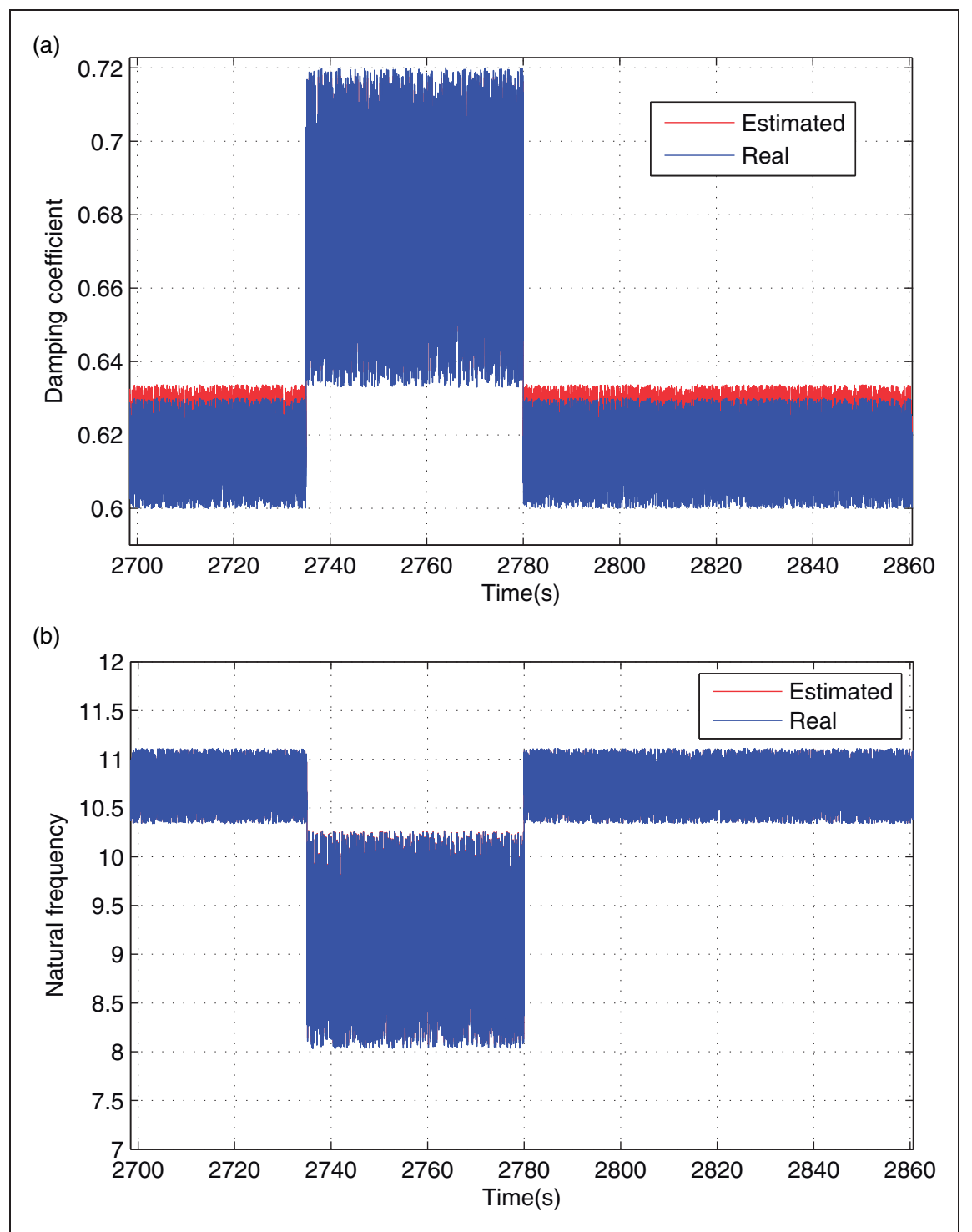

Figure 7. Parameters estimation. (a) Control angle, (b) Pitch angle, (c) Error with FTC.

and the error signal calculated between the two signal are given in Figure 3.

Figure 3 shows that the real pitch angle is far away from the desired one at the time when the fault occurs. To solve this problem a control reconfiguration is required. Using Algorithm 3, the reconfiguration constitutes the activation of virtual sensor or actuator according to the information provided by the FDI module. This FDI procedure requires the residual signal in this case of fault. The activated residuals are presented in Figure 4.

A residual evaluation unit is used to compare the residuals with the threshold to generate the observed fault signature presented in Figure 5 that allows the isolation of the fault using the fault signature matrix corresponding to the previous set of residuals presented in Table 3. The residuals are in rows and the previous fault scenarios in columns. 

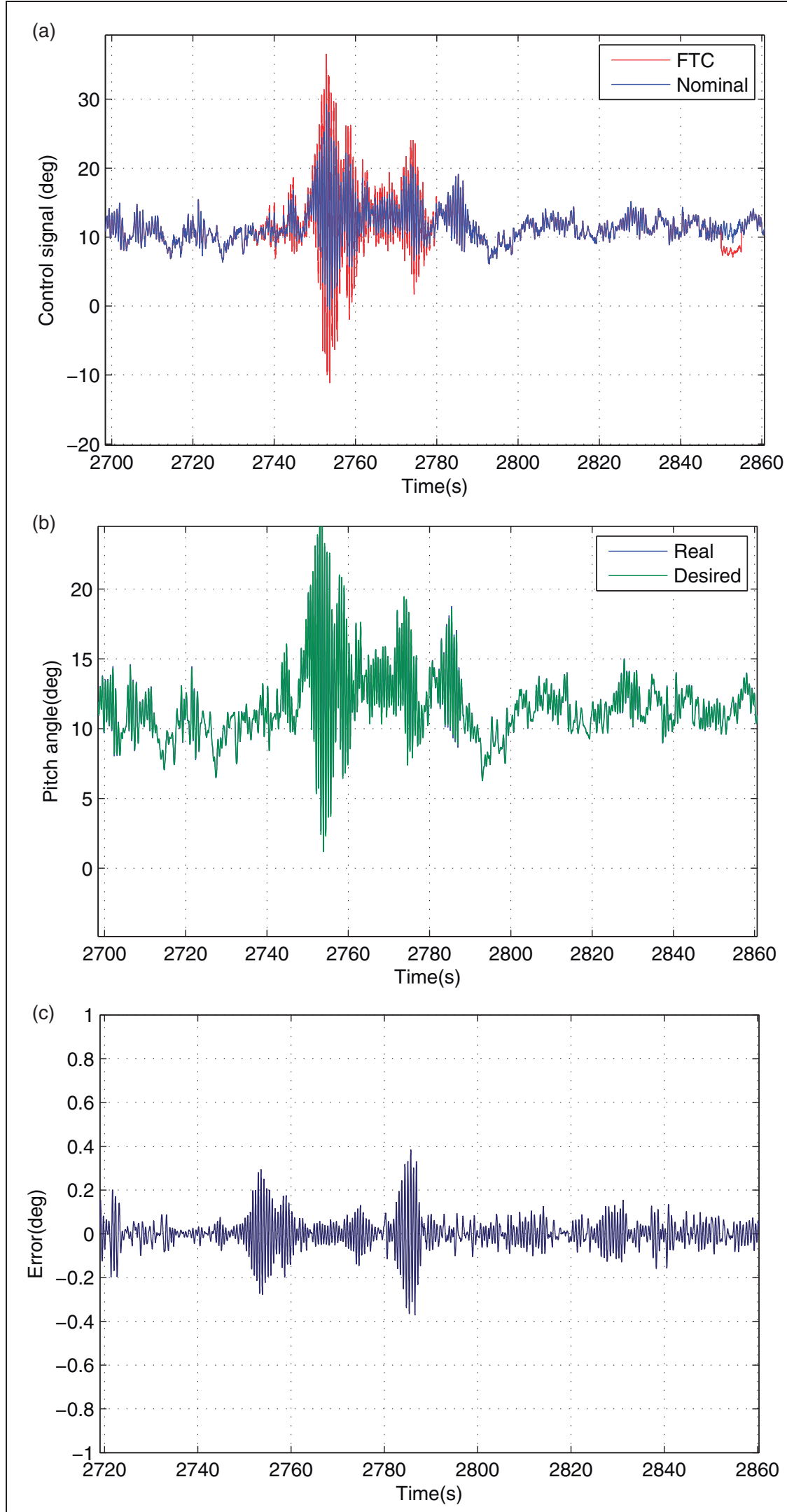

Figure 8. System responses with FTC. 
The fault indicators are presented in Figure 6.

The fault tolerance in the actuator fault scenario requires identifying the fault damping ratio and natural frequency. Using the identification approach described in Section 3, these parameters are obtained and presented in Figure 7.

The new FTC signal in this fault scenario, the real and the desired pitch angle and the error between the two pitch angle are presented in Figure 8.

\section{Comparison with other works}

The FDI/FTC wind turbine benchmark used in this work is largely treated in the literature as e.g. in literature. ${ }^{37-39}$

- In Sanchez et al. ${ }^{37}$ (ATD1), the majority of faults are well detected except the actuator fault that is a change in the pitch system dynamics $\left(f_{8}\right)$. However, this fault is detected using the approach proposed in this paper.

- In Sheibat-Othman et al. ${ }^{38}$ (ATD2), only $f_{1}, f_{6}$ and $f_{7}$ are detected with a time larger than the required one.

- In Zeng et al. ${ }^{39}$ (ATD3), all faults are detected but for example $f_{1}$ and $f_{10}$ are detected after a large time period which it is improved using the approach proposed in this paper. This comparison is summarized in Table 4.

In this table $N C$ denotes not considered, $A T D$ means the time detection with the approach proposed in this work and TD1,TD2,TD3 indicate the time detection with the fault detection methods ATD1, ATD2 and ATD3 proposed respectively in literature. $^{37-39}$

\section{Conclusion}

In this paper, an integrated FDI/FTC approach for a wind turbine benchmark has been proposed. FDI is based on a robust fault detection approach combined with a subspace LPV estimation algorithm. Fault isolation is based on an algorithm that combines the column/row matching of the observed fault signature with the theoretical ones. FTC is addressed using virtual sensors/actuators and subspace fault identification. Satisfactory results have been obtained for both FDI and the FTC in several fault scenarios proposed in the considered wind turbine benchmark. In the case of the pitch system, an actuator fault which corresponds to dynamic change in the pitch parameters and a sensor fault consisting of a fixed pitch angle value. Future research is focused on the validating of the fault tolerant control procedure of faults using the second wind turbine benchmark that simulates the wind turbine using a high fidelity simulator (FAST).

\section{Declaration of conflicting interests}

The author(s) declared no potential conflicts of interest with respect to the research, authorship, and/or publication of this article.

\section{Funding}

The author(s) received no financial support for the research, authorship, and/or publication of this article.

\section{References}

1. El Bakri M and Boumhidi I. Fuzzy model-based faults diagnosis of the wind turbine benchmark. Proc Comput Sci 2018; 127: 464-470.

2. Ben Djoudi HC, Hafaifa A, Djoudi D, et al. Fault tolerant control of wind turbine via identified fuzzy models prototypes. Diagnostyka 2020; 21: 3-13.

3. Zhang X. Fault detection and isolation of the wind turbine benchmark: an estimating approach. In: 18th World Congress of the International Federation of Automatic Control, Milan, Italy, 28 August-2 September 2011.

4. Laouti N and Othman S. Support vector machines for fault detection in wind turbines. In: 18th World Congress of the International Federation of Automatic Control, Milan, Italy, 28 August-2 September 2011.

5. Montes de Oca S, Tornil-Sin S, Puig V, et al. Faulttolerant control design using the linear parameter varying approach. Int J Robust Nonlinear Control 2014; 24: 1969-1988.

6. Sloth C, Esbensen T and Stoustrup J. Robust and faulttolerant linear parameter-varying control of wind turbines. Mechatronics 2011; 21: 645-659.

7. Jun $H$, Yang G and Ye D. Switching fault-tolerant control for a class of nonlinear systems with actuator faults. In: 25th Chinese control and decision conference (CCDC), Guiyang, China, 25-27 May 2013.

8. Blanke M, Kinnaert $\mathrm{M}$ and Lunze J. Diagnosis and fault-tolerant control. Berlin Heidelberg: SpringerVerlag, 2016.

9. Boussaid B, Aubrun C and Abdelkrim MN. Two-level active fault tolerant control approach. In: The eighth international multi-conference on systems, signals devices, Sousse, Tunisia, 22-25 March 2011.

10. Odgaard P and Stoustrup J. Orthogonal bases used for feed forward control of wind turbines. In: 18th World Congress of the International Federation of Automatic Control, Milan, Italy, 28 August-2 September 2011.

11. Simani S and Castaldi P. Active actuator fault-tolerant control of a wind turbine benchmark model. Int $J$ Robust Nonlinear Control 2014; 24: 1283-1303.

12. Rothenhagen K and Fuchs F. Current sensor fault detection and reconfiguration for a doubly fed induction generator. In: Power electronics specialists conference, 2007 (PESC 2007). Piscataway: IEEE, pp. 27322738.

13. Poure P, Weber P, Theilliol D, et al. Fault-tolerant power electronic converters: reliability analysis of active power filter. In: IEEE international symposium 
on industrial electronics, 2007. ISIE 2007. Piscataway: IEEE, pp.3174-3179.

14. Boussaid B, Aubrun C and Abdelkrim MN. Active fault tolerant approach for wind turbines. In: The 2011 International conference on communications, computing and control applications (CCCA'11), Hammamet, Tunisia, 3-5 March 2011.

15. Boussaid B, Aubrun C and Abdelkrim MN. Set-point reconfiguration approach for the FTC of wind turbines. In: 18th World Congress of the International Federation of Automatic Control, Milan, Italy, 28 August-2 September 2011.

16. Sami M and Patton R. Fault tolerant adaptive sliding mode controller for wind turbine power maximisation. In Proceedings of the 7th IFAC Symposium on Robust Control Design The International Federation of Automatic Control Aalborg, Denmark, volume 7. pp. 499-504.

17. Sami M and Patton R. Wind turbine power maximisation based on adaptive sensor fault tolerant sliding mode control. In: 20th Mediterranean conference on control \& automation (MED), 2012. Piscataway: IEEE, pp.1183-1188.

18. Badihi H, Zhang Y and Hong H. Fuzzy gain-scheduled active fault-tolerant control of a wind turbine. J Franklin Inst 2014; 351: 3677-3706.

19. Kamal E, Aitouche A, Ghorbani R, et al. Robust fuzzy fault-tolerant control of wind energy conversion systems subject to sensor faults. IEEE Trans Sustain Energy 2012; 3: 231-241.

20. Shamma J. Control of linear parameter varying systems with applications. Boston, MA: Springer. 2012.

21. Odgaard PF and Stoustrup J. A benchmark evaluation of fault tolerant wind turbine control concepts. IEEE Trans Contr Syst Technol 2015; 23: 1221-1228.

22. Odgaard P, Stoustrup J and Kinnaert M. Fault tolerant control of wind turbines - a benchmark model. IEEE Trans Contr Syst Technol 2013; 21: 1168-1182.

23. Niss M, Esbensen T and Sloth C. A Youla-Kucera approach to gain-scheduling with application to wind turbine control. In: 18th IEEE international conference on control applications, Saint Petersburg, Russia, 8-10 July 2009.

24. Van Willem J and Verhagen M. Subspace identification of bilinear and LPV systems for open-and closed-loop data. Automatica 2009; 45: 371-381.

25. Puig V. Fault diagnosis and fault tolerant control using set-membership approaches: application to real case studies. Int J Appl Math Comput Sci 2010; 20: 619-635.

26. Gebraad P, van Wingerden J, van der Veen $G$, et al. LPV subspace identification using a novel nuclear norm regularization method. In: American control conference on O'Farrell Street, San Francisco, CA, USA, 29 June1 July 2010.

27. Ljung L. System Identification: Theory for the User. Prentice Hall information and system sciences series, Prentice Hall PTR, 1999.

28. Van Willem $\mathbf{J}$ and Verhagen M. Subspace identification of MIMO LPV systems: the PBSID approach. In: Proceedings of the 47th IEEE conference on decision and control, Cancun, Mexico, 9-11 December 2008.

29. Verdult V and Verhaegen M. Kernel methods for subspace identification of multivariable LPV and bilinear systems. Automatica 2005; 41: 1557-1565.

30. Odgaard P and Stoustrup J. Fault tolerant control of wind turbines - a benchmark model. In: 7th IFAC symposium on fault detection, supervision and safety of technical processes, Barcelona, Spain, 30 June-3 July 2009.

31. Kamal E and Aitouche A. Robust fault tolerant control of DFIG wind energy systems with unknown inputs. Renewable Energy 2013; 56: 2-15.

32. Hallouzi R, Verdult V, Babuska R, et al. Fault detection and identification of actuator faults using linear parameter varying models. In: Proceedings of the 16th IFAC world congress, Prague, Czech Republic, 3-8 July 2005.

33. Rodrigues M, Theilliol D, Aberkane S, et al. Fault tolerant control design for polytopic LPV systems. Int $J$ Appl Math Comput Sci 2007; 17: 27-37.

34. Patton R. LPV fault estimation and FTC of a two-link manipulator. In: American control conference Marriott Waterfront, Baltimore, MD, USA, 30 June-2 July 2010.

35. de Oca S, Puig V, Witczak M, et al. Fault-tolerant control strategy for actuator faults using LPV techniques: application to a two degree of freedom helicopter. Int $J$ Appl Math Comput Sci 2012; 22: 161-171.

36. Richter JH, Heemels WPMH, van de Wouw N, et al. Reconfigurable control of piecewise affine systems with actuator and sensor faults: stability and tracking. Automatica 2011; 47: 678-691.

37. Sanchez H, Escobet T, Puig V, et al. Fault diagnosis of an advanced wind turbine benchmark using intervalbased ARRS and observers. IEEE Trans Ind Electron 2015; 62: 3783-3793.

38. Sheibat-Othman N, Othman S, et al. Fault detection and isolation in wind turbines using support vector machines and observers. In: American control conference (ACC), 2013. Piscataway: IEEE, pp.4459-4464.

39. Zeng J, Lu D, Zhao Y, et al. Wind turbine fault detection and isolation using support vector machine and a residual-based method. In: American control conference (ACC), 2013. Piscataway: IEEE, pp.3661-3666. 